\title{
O Acidente Vascular Encefálico como complicação neurológica da COVID-19
}

\author{
Stroke as a neurological complication of COVID-19 \\ Accidente cerebrovascular como complicación neurológica de COVID-19
}

Recebido: 27/12/2020 | Revisado: 03/01/2021 | Aceito: 06/01/2021 | Publicado: 07/01/2021

Ianka Heloisa Alencar Santos

ORCID: https://orcid.org/0000-0002-2057-0727 Universidade Tiradentes, Brasil

E-mail: iankaheloisa@outlook.com

Sávio Menezes Farias

ORCID: https://orcid.org/0000-0002-1727-9971 Universidade Tiradentes, Brasil

E-mail: savio.menezes@souunit.com.br

Thandara Rejane Santos Ferreira Andrade

ORCID: https://orcid.org/0000-0002-3385-7727 Universidade Tiradentes, Brasil

E-mail: thandara456@outlook.com

Grayce Ellen Souza Rezende

ORCID: https://orcid.org/0000-0002-6197-5720 Universidade Tiradentes, Brasil

E-mail: grayce-ellen@ hotmail.com

Erivelton Cunha Torres

ORCID: https://orcid.org/0000-0002-3866-6305 Universidade Tiradentes, Brasil

E-mail: eriveltontorres18@gmail.com

Anderson Batista Cavalcante

ORCID: https://orcid.org/0000-0002-4168-4379 Pontifícia Universidade Católica de Campinas, Brasil E-mail: meiro1976@hotmail.com

Carla Viviane Freitas de Jesus ORCID: https://orcid.org/0000-0002-7775-6610 Universidade Tiradentes, Brasil E-mail: carlavfj@gmail.com

Daniele Martins de Lima Oliveira ORCID: https://orcid.org/0000-0003-2838-0204 Universidade Tiradentes, Brasil

E-mail: daniele.martins@souunit.com.br

Yasmim Anayr Costa Ferrari

ORCID: https://orcid.org/0000-0003-1766-341X Universidade Tiradentes, Brasil

E-mail: yasmimanayr@hotmail.com

Dênison Pereira da Silva

ORCID: https://orcid.org/0000-0001-7038-6496 Universidade Tiradentes, Brasil

E-mail: denisonbm@yahoo.com.br

\begin{abstract}
Resumo
Objetivo: Analisar o Acidente Vascular Encefálico como complicação causada pela COVID-19, bem como suas repercussões clínicas. Metodologia: Trata-se de uma revisão integrativa de artigos contendo informações de diagnósticos de sinais e sintomas neurológicos. A busca dos artigos científicos foi realizada nas seguintes bases de dados: Literatura Latino-americana e do Caribe em Ciências da Saúde (LILACS), SCIENCE DIRECT, Medical Literature Analysis and Retrieval System Online (MEDLINE) e Scientific Electronic Library Online (SCIELO). Resultados: Foram selecionados sete artigos para realização da revisão integrativa. Todos os manuscritos foram publicados em 2020 (100\%). Foram elencadas informações referentes aos autores, país, título, objetivo e resultados encontrados nos estudos, elaborando discussões acerca de cada artigo. Em contrapartida, os autores mostram que o SARS-CoV-2 afeta muito além dos pulmões e é mais do que uma síndrome gripal, demonstrando caráter multissistêmico, independente de sexo, idade e comorbidades crônicas. Conclusão: A revisão realizada mostrou que existe uma tendência que pacientes internados com sintomas graves de COVID-19 estejam mais propensos a desenvolverem problemas neurológicos como o AVE. No entanto, mais estudos são necessários que contenham uma qualidade da evidência superior, bem como, que os profissionais de saúde realizem um diagnóstico mais amplo capaz de abranger todos os sinais e sintomas presentes em pacientes com coronavírus.
\end{abstract}

Palavras-chave: Acidente vascular cerebral; Tromboembolia; Infecções por coronavirus; COVID-19. 


\begin{abstract}
Objective: To analyze stroke as a complication caused by COVID-19, as well as its clinical repercussions. Methodology: This is an integrative review of articles containing information on diagnoses of neurological signs and symptoms. The search for scientific articles was carried out in the following databases: Latin American and Caribbean Literature on Health Sciences (LILACS), SCIENCE DIRECT, Medical Literature Analysis and Retrieval System Online (MEDLINE) and Scientific Electronic Library Online (SCIELO). Results: Seven articles were selected to perform the integrative review. All manuscripts were published in 2020 (100\%). Information was collected regarding the authors, country, title, objective and results found in the studies, elaborating discussions about each article. On the other hand, the authors show that SARS-CoV-2 affects far beyond the lungs and is more than a flu syndrome, demonstrating a multisystemic character, regardless of gender, age and chronic comorbidities. Conclusion: The review showed that there is a tendency that hospitalized patients with severe symptoms of COVID-19 are more likely to develop neurological problems such as a veactually. However, more studies are needed to contain a higher quality of evidence, as well as that health professionals make a broader diagnosis capable of covering all signs and symptoms present in patients with coronavirus.
\end{abstract}

Keywords: Stroke; Thromboembolism; Coronavirus infections; COVID-19.

\begin{abstract}
Resumen
Objetivo: Analizar el ictus como una complicación causada por COVID-19, así como sus repercusiones clínicas. Metodología: Esta es una revisión integradora de artículos que contienen información sobre diagnósticos de signos y síntomas neurológicos. La búsqueda de artículos científicos se llevó a cabo en las siguientes bases de datos: Literatura Latinoamericana y del Caribe sobre Ciencias de la Salud (LILACS), SCIENCE DIRECT, Medical Literature Analysis and Retrieval System Online (MEDLINE) y Scientific Electronic Library Online (SCIELO). Resultados: Se seleccionaron siete artículos para realizar la revisión integradora. Todos los manuscritos fueron publicados en 2020 (100\%). Se recopiló información sobre los autores, país, título, objetivo y resultados encontrados en los estudios, elaborando discusiones sobre cada artículo. Por otro lado, los autores muestran que el SARS-CoV-2 afecta mucho más allá de los pulmones y es más que un síndrome de gripe, demostrando un carácter multisistémico, independientemente del género, la edad y las comorbilidades crónicas. Conclusión: La revisión mostró que existe una tendencia a que los pacientes hospitalizados con síntomas graves de COVID-19 son más propensos a desarrollar problemas neurológicos como una veamáticamente. Sin embargo, se necesitan más estudios para contener una mayor calidad de la evidencia, así como que los profesionales de la salud hacen un diagnóstico más amplio capaz de cubrir todos los signos y síntomas presentes en pacientes con coronavirus.
\end{abstract}

Palabras clave: Accidente cerebrovascular; Tromboembolia; Infecciones por coronavirus; COVID-19.

\title{
1. Introdução
}

Em 11 de março de 2020, a Organização Mundial da Saúde (OMS) declarou pandemia para a infecção por SARSCoV-2, o novo coronavírus causador da COVID-19, se caracteriza como um dos grandes entraves da saúde pública global, com manifestações clínicas que variam desde febre, dispneia, tosse e fadiga até complicações críticas tais como, síndrome respiratória aguda grave, lesões cardíacas e renais, sepse, coagulações intravasculares e distúrbios metabólicos (Lin et al., 2020; Nepal et al., 2020). Em 16 de setembro de 2020, no mundo já houveram mais de 29 milhões de casos e 926.544 mortes (Opas, 2020). O Brasil como um dos países mais afetados, possui 4 milhões de casos e 133.119 óbitos (Brasil, 2020).

As manifestações neurológicas são frequentes na COVID-19, com incidência de 36\% e aumentam na forma grave da enfermidade. O SARS-CoV-2 possui afinidade com a enzima conversora de angiotensina 2 (ECA2) presente no trato respiratório, rins, fígado, intestino delgado e células do sistema imunológico (Al-ani et al., 2020). Ademais, foi pressuposto que o vírus se localiza no Sistema Nervoso Central (SNC) e a disseminação para a área nervosa se dá por via hematogênica, linfática ou transneuronal (Ferrarese et al., 2020; Leonardi et al., 2020). O Acidente Vascular Encefálico (AVE) tem incidência de 3,7 a 5\% nos pacientes acometidos pela COVID-19. Sendo um agravo com elevados índices de morbimortalidade e déficits, que atrelado a infecção com coronavírus contribui para um prognóstico desfavorável ao paciente (Ortiz et al., 2020; Whittaker et al., 2020). Diante da problemática exposta, emergiu a seguinte questão: O que se sugere nas produções cientificas quanto o AVE e os eventos trombóticos na COVID-19 e as implicações clínicas aos pacientes acometidos por tal agravo? Nesta perspectiva, o objetivo do presente estudo consiste em analisar o Acidente Vascular Encefálico como complicação causada pela COVID-19, bem como suas repercussões clínicas.

O presente trabalho foi proposto em decorrência da pandemia de COVID-19. É de fundamental relevância, o 
conhecimento acerca do vírus SARS-CoV-2 e suas diversas manifestações e eventos trombóticos que se caracterizam como fatores danosos no prognóstico dos pacientes acometidos por tal afecção. O AVE é uma das emergências médicas mais preocupantes, com elevadas taxas de incidência e prevalência na população, além de, provocar inúmeras incapacidades e distúrbios na qualidade de vida dos indivíduos. Assim, espera-se contribuir para a ampliação de informações acerca da relação do AVE e da COVID-19, bem como o conhecimento da fisiopatologia, mecanismos virais e clínicos nos indivíduos com tal afecção.

\section{Metodologia}

Trata-se de uma pesquisa documental através da Revisão Integrativa (RI) da literatura, realizada por meio de levantamento bibliográfico a partir de dados secundários, desenvolvida entre os meses de setembro a outubro de 2020 . A revisão integrativa agrupa e sintetiza resultados de pesquisas anteriores, compreende os estudos relacionados a questão norteadora que orienta a busca desta literatura. As evidências científicas resumidas e analisadas permitem a obtenção de conclusões gerais sobre o problema da pesquisa. Por conseguinte, o processo de construção da revisão integrativa segue basicamente cinco etapas: formulação do problema, coleta de dados sobre a busca da literatura, avaliação dos dados, análise dos dados, apresentação e interpretação dos resultados. O presente estudo possui caráter qualitativo, visto que contempla a análise e apontamento por intermédio dos pesquisadores (Crossetti, 2012; Pereira et al., 2018).

A busca dos artigos científicos foi realizada nas seguintes bases de dados: Literatura Latino-americana e do Caribe em Ciências da Saúde (LILACS), SCIENCE DIRECT, Medical Literature Analysis and Retrieval System Online (MEDLINE) e Scientific Electronic Library Online (SCIELO). Foram utilizados os Descritores em Ciências da Saúde (DeCS): Stroke, Thromboembolism e Coronavirus Infections, combinados pelo operador booleano AND. Os descritores foram combinados para abranger a busca, culminando em duas associações: "Stroke AND Coronavirus Infections ” e Thromboembolism AND Coronavirus Infections".

Os critérios de inclusão adotados foram: artigos disponíveis na íntegra nos idiomas português, inglês e espanhol; artigos originais e revisões sistemáticas; publicados entre os meses de janeiro a setembro de 2020; com metodologia coesa e bem delineada. Foram excluídos relatos de casos, teses, dissertações, duplicidade nas bases de dados pesquisadas e estudos que não atendessem a temática proposta.

Para elaboração do presente estudo foram instituídas algumas etapas: delimitação do tema e elaboração da pergunta norteadora; definição dos critérios de seleção dos estudos; análise, avaliação e seleção dos estudos incluídos na produção; extração, discussão e síntese dos resultados encontrados. O fluxograma (Figura 1) descreve as etapas da coleta de dados. 
Figura 1 - Etapa de coleta de dados. Aracaju-SE, Brasil, 2020.

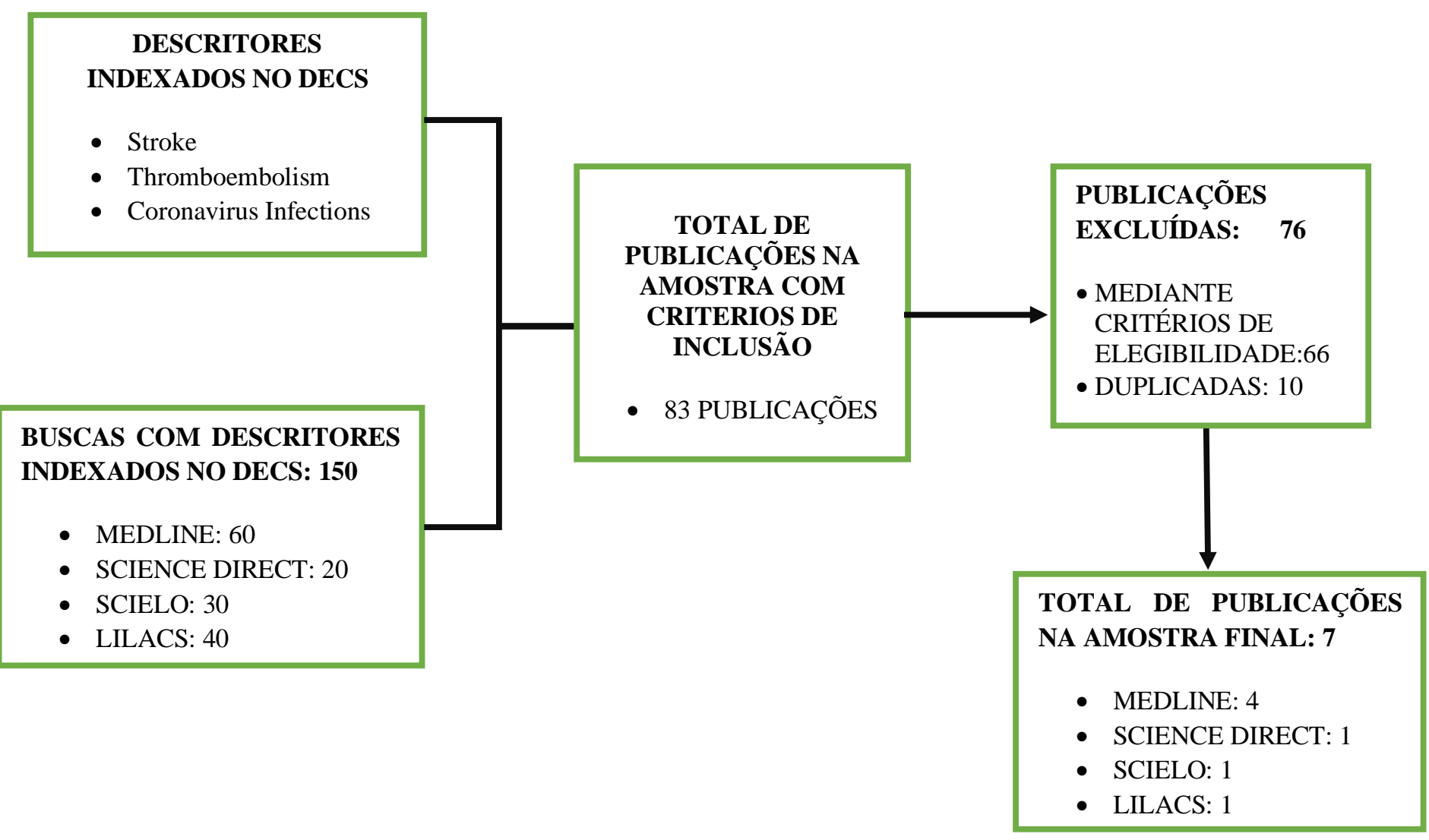

Fonte: Autores.

Conforme a Figura 1, através das buscas por meio dos descritores foram encontrados 150 artigos. Por conseguinte, foram aplicados os critérios de inclusão, resultando na seleção de 83 artigos para avaliação dos títulos e resumos. Após a análise, artigos foram selecionados para a leitura na íntegra. A amostra final foi composta por 07 artigos, visto que esses seguiam os critérios de elegibilidade antepostos para a pesquisa.

Por se tratar de dados secundários, não foi necessária a submissão ao Comitê de Ética em Pesquisa. Contudo, obedecendo os princípios éticos, todos os trabalhos utilizados para a confecção deste artigo foram citados.

\section{Resultados}

Foram selecionados sete artigos para realização da revisão integrativa. Todos os manuscritos foram publicados em 2020 (100\%). Na Tabela 1 encontram-se informações referentes aos autores, país, título, objetivo e resultados encontrados nos estudos. 
Tabela 1 - Descrição dos artigos selecionados de acordo com autores, país, título, objetivo resultados. Aracaju-SE, Brasil, 2020 .

\begin{tabular}{|c|c|c|c|c|}
\hline AUTORES & PAÍS & TÍTULO & OBJETIVO & RESULTADOS \\
\hline $\begin{array}{c}\text { Ortiz, M. et } \\
\text { al. }\end{array}$ & Colombia & $\begin{array}{l}\text { ACV y covid-19: } \\
\text { una revisión de los } \\
\text { estudios } \\
\text { observacionales } \\
\text { publicados en época } \\
\text { de pandemia }\end{array}$ & $\begin{array}{c}\text { Conhecer a } \\
\text { experiência de } \\
\text { diferentes partes do } \\
\text { mundo em relação ao } \\
\text { AVE e a COVID-19, } \\
\text { a fim de melhorar o } \\
\text { reconhecimento e o } \\
\text { manejo clínico de } \\
\text { tais agravos. }\end{array}$ & $\begin{array}{c}\text { Os estados } \\
\text { inflamatórios e de } \\
\text { hipercoagulação que se } \\
\text { apresentam na COVID- } \\
\text { 19, provavelmente se } \\
\text { relacionam com o AVE, } \\
\text { causando elevados } \\
\text { números de oclusões de } \\
\text { grandes vasos. }\end{array}$ \\
\hline $\begin{array}{c}\text { Nepal, G. et } \\
\text { al. }\end{array}$ & Nepal & $\begin{array}{c}\text { Neurological } \\
\text { manifestations of } \\
\text { COVID-19: a } \\
\text { systematic review }\end{array}$ & $\begin{array}{c}\text { Apresentar uma } \\
\text { análise sistemática } \\
\text { das manifestações } \\
\text { neurológicas } \\
\text { apresentadas pelos } \\
\text { pacientes. }\end{array}$ & $\begin{array}{c}\text { Raramente a COVID-19 } \\
\text { pode apresentar } \\
\text { manifestações no } \\
\text { sistema nervoso central, } \\
\text { como o AVE } \\
\text { isquêmico. }\end{array}$ \\
\hline Tan, Y. et al. & Singapura & $\begin{array}{l}\text { COVID-19 and } \\
\text { ischemic stroke: a } \\
\text { systematic review } \\
\text { and meta-summary } \\
\text { of the literature }\end{array}$ & $\begin{array}{c}\text { Evidenciar as } \\
\text { características } \\
\text { clínicas, achados de } \\
\text { neuroimagem e } \\
\text { resultados de AVE } \\
\text { isquêmico agudo em } \\
\text { pacientes com } \\
\text { COVID-19. }\end{array}$ & $\begin{array}{l}\text { A incidência do AVE } \\
\text { isquêmico em pacientes } \\
\text { com COVID-19 é de } \\
\text { 1,2\%, com uma alta } \\
\text { taxa de mortalidade. D- } \\
\text { dímero elevado e } \\
\text { fibrinogênio parecem } \\
\text { ser proeminentes nos } \\
\text { pacientes. }\end{array}$ \\
\hline
\end{tabular}




\begin{tabular}{|c|c|c|c|c|}
\hline $\begin{array}{c}\text { Ghannan et } \\
\text { al. }\end{array}$ & Alemanha & $\begin{array}{c}\text { Neurological } \\
\text { involvement of } \\
\text { coronavírus disease } \\
\text { 2019: a systematic } \\
\text { review }\end{array}$ & $\begin{array}{c}\text { Investigar a } \\
\text { associação de } \\
\text { pacientes infectados } \\
\text { pela COVID-19 com } \\
\text { distúrbios } \\
\text { neurológicos. }\end{array}$ & $\begin{array}{c}\text { Dentre os problemas } \\
\text { neurológicos citados, os } \\
\text { mais relatados foram } \\
\text { Acidente vascular } \\
\text { cerebral de grande } \\
\text { porte, síndrome de } \\
\text { Guillain-Barre e } \\
\text { meningocefalite. }\end{array}$ \\
\hline Violi et al. & Itália & $\begin{array}{c}\text { Hypercoagulation } \\
\text { and antithrombotic } \\
\text { treatment in } \\
\text { coronavirus 2019: a } \\
\text { new challenge }\end{array}$ & $\begin{array}{c}\text { Avaliar pacientes } \\
\text { com a COVID-19 } \\
\text { em estágio grave } \\
\text { com disfunções } \\
\text { arteriais, AVE } \\
\text { isquêmico e } \\
\text { hipercoagulação. }\end{array}$ & $\begin{array}{c}\text { Pacientes em estado } \\
\text { grave tem mais chances } \\
\text { de desencadearem } \\
\text { problemas de } \\
\text { coagulação. Assim a } \\
\text { associação entre } \\
\text { trombose, AVE, e } \\
\text { problemas arteriais deve } \\
\text { ser considerada. }\end{array}$ \\
\hline $\begin{array}{c}\text { Montalvan et } \\
\text { al. }\end{array}$ & $\begin{array}{l}\text { Estados } \\
\text { Unidos }\end{array}$ & $\begin{array}{c}\text { Neurological } \\
\text { manifestations of } \\
\text { covid-19 and other } \\
\text { coronavirus } \\
\text { infections: a } \\
\text { systematic review }\end{array}$ & $\begin{array}{c}\text { Analisar a relação } \\
\text { entre o SARS-CoV-2 } \\
\text { e sintomas } \\
\text { neurológicos que } \\
\text { acometeram } \\
\text { pacientes em estado } \\
\text { grave e formular os } \\
\text { mecanismos virais } \\
\text { no sistema nervoso. }\end{array}$ & $\begin{array}{l}\text { O AVE está relacionado } \\
\text { ao SARS-CoV-2 por } \\
\text { hipercoagulabilidade, } \\
\text { arterite e disfunção } \\
\text { endotelial, no entanto o } \\
\text { mecanismo ainda precisa } \\
\text { ser explorado. }\end{array}$ \\
\hline Pranata et al. & Indonésia & $\begin{array}{c}\text { Impact of } \\
\text { cerebrovascular and } \\
\text { cardiovascular } \\
\text { diseases on mortality } \\
\text { and severity of } \\
\text { covid-19-systematic } \\
\text { review, meta- } \\
\text { analysis, and meta- } \\
\text { regression }\end{array}$ & $\begin{array}{c}\text { Avaliar a relação } \\
\text { entre doenças } \\
\text { cardiovasculares e } \\
\text { cerebrovasculares em } \\
\text { pacientes de } \\
\text { COVID-19 } \\
\text { internados em estado } \\
\text { grave. }\end{array}$ & $\begin{array}{c}\text { Doenças } \\
\text { cerebrovasculares e } \\
\text { cardiovasculares levam a } \\
\text { maiores chances de morte } \\
\text { e de piora do quadro } \\
\text { clínico nos pacientes. } \\
\text { Essa ligação se deve ao } \\
\text { fato de que o vírus } \\
\text { diminui a expressão de } \\
\text { ECA2, uma enzima } \\
\text { presente no tecido } \\
\text { respiratório e no tecido } \\
\text { cardíaco. }\end{array}$ \\
\hline
\end{tabular}


A Tabela 1 demonstra a realização de investigações em diversas partes do território global. No tocante ao objetivo dos estudos, foi notável entre os mesmo a avaliação das principais manifestações neurológicas desenvolvidas pelos pacientes com a infecção por SARS-CoV-2. Em relação aos resultados, os manuscritos corroboram que o patógeno provoca danos ao sistema nervoso, ocasionando complicações neurológicas, tais como o AVE, que por sua vez desenvolve um agravamento no quadro clínico do paciente, motivando um prognóstico desfavorável aos indivíduos afetados.

\section{Discussão}

A partir do estudo de Ortiz e colaboradores (2020), foi realizada uma descrição das características clínicas de 43 pacientes com a infecção por COVID-19 e o Acidente Vascular Encefálico como complicação neurológica. A média de idade dos indivíduos foi entre 67 e 70 anos; quanto aos mecanismos virais foi notório a hiperatividade do sistema inflamatório e alterações na coagulação, a elevação dos valores do D-dímero, Fibrinogênio, PCR e Ferritina foram achados no estudo. Sendo estes, marcadores de inflamação e coagulação, a resposta inflamatória exacerbada provocada pelo SARS-CoV-2 gera aumento na coagulação e acelera a ruptura de placas ateroscleróticas, além de induzir fatores de coagulação que provocam a isquemia ou trombose.

O estudo de Nepal et al. (2020), reúne as principais manifestações neurológicas presentes nos pacientes acometidos pela COVID-19. Em um grupo de 214 pacientes em um estudo observacional em Wuhan, o AVE foi desenvolvido em 6 $(2,8 \%)$ indivíduos, dentre os mesmos, 5 pacientes possuíram a apresentação mais grave da COVID-19. Outrossim, idade avançada e comorbidades, tais como: Hipertensão arterial sistêmica (HAS), Diabetes mellitus (DM) e Doenças cerebrovasculares anteriores, foram achados comuns entre os pacientes. Sugere-se que o AVE isquêmico manifesta-se a partir de uma tempestade de citocinas que acarreta danos endoteliais e coagulação intravascular disseminada, elevando o risco de trombose e ocasionando assim a enfermidade.

Tan et al. (2020), relata que a incidência do AVE entre os pacientes com COVID-19 varia entre 0,9\% a 2,7\%. Ademais, o estudo demonstra uma elevada taxa de mortalidade de $38 \%$ reportada entre os casos. Em um grupo 4466 pacientes com COVID-19, 135 possuíam o AVE, além de que em sua maioria eram homens (62,3\%), com média de idade de 63.4 anos; no tocante as comorbidades mais comuns entre os pacientes, se incluíam: Hipertensão arterial sistêmica (64,5\%), Diabetes Mellitus (42,6\%) e Dislipidemias (32\%). Em relação a manifestação do AVE nos pacientes foi considerada de moderada a grave, com elevada prevalência de trombose, embolia e estenose de vasos.

Para Ghannan et al. (2020), o Acidente Vascular Encefálico isquêmico foi a manifestação neurológica mais comum, sucedendo em 42,7\% dos indivíduos. Sendo evidenciado, através de estudo com 388 pacientes com COVID-19 na Itália, dentre os quais $21 \%$ sofreram eventos trombóticos, tais como: AVE isquêmico, tromboembolismo venoso e síndrome coronariana aguda. Em relação ao mecanismo viral, o SARS-Cov-2 liga-se a enzima conversora de angiotensina 2 (ECA2) nas células endoteliais, o que promove ação pró-inflamatória e vasoconstritora contribuindo em efeitos danosos aos pacientes.

Segundo Violi et al. (2020) uma vez que o agente etiológico atinge as células do tecido pulmonar, com o surgimento de um quadro clínico grave semelhante a uma pneumonia, é necessário que o médico e o enfermeiro procurem investigar nos pacientes sinais e sintomas que possam indicar coagulação ativa. Sendo a mesma constatada em decorrência do quadro grave da COVID-19, dessa forma, o tratamento trombolítico é uma opção.

De acordo com Pranata et al. (2020) quando se decretou a pandemia em março, a COVID-19 era classificada como uma doença de caráter respiratório, como uma gripe que afetava principalmente a população idosa e com comorbidades préexistentes. Em contrapartida, os autores mostram que o SARS-CoV-2 afeta muito além dos pulmões e é mais do que uma síndrome gripal, demonstrando caráter multissistêmico. Por conseguinte, é possível o acometimento de danos no coração, 
cérebro e rins, além de desencadear complicações de hipercoagulação, trombose, AVE e parada cardíaca, independente de sexo, idade, e doenças como hipertensão e diabetes.

O estudo de Montalvan et al. (2020) demonstra que os pacientes com históricos ou fatores de risco vasculares, possuem maior probabilidade de desenvolver o AVE com complicações como hipotensão, insuficiência cardíaca, e choque, que contribuem na hipoperfusão, mecanismos embólicos do AVE e oclusão de grandes vasos. No estudo observacional na China com 191 pacientes, 23\% sofreram complicações com insuficiência cardíaca, 20\% com choque séptico, 19\% com coagulopatia e 17\% com lesão cardíaca aguda, sendo condições que predispõem ao AVE. Faz-se necessário maiores investigações no tocante aos fatores virais que causam hipercoagulabilidade e disfunção endotelial, visto que podem acarretar no desenvolvimento do AVE isquêmico ou sangramento cerebral.

Na presente revisão integrativa observou-se limitações e dificuldades. Destaca-se o fato de ser uma temática nova e pouco discutida entre os pesquisadores, dessa forma não haviam muitos manuscritos disponíveis e que se relacionassem ao tema em questão. Ademais, restrições de idiomas podem ter afetado a identificação de casos ou resultados relevantes, pois muitos dos estudos eram em inglês, porém possuíam dados de pacientes publicados em seus idiomas regionais, como o chinês e o italiano.

\section{Conclusão}

O Acidente Vascular Encefálico é uma complicação neurológica proveniente da infecção por SARS-CoV-2. Em síntese, foi evidenciado que pacientes de idade avançada, sexo masculino e com presença de comorbidades foram os mais afetados. Ademais, é importante ressaltar que alterações no D-dímero, Fibrinogênio, PCR, Ferritina e outros marcadores de inflamação e coagulação foram achados encontrados nos estudos. Portanto, é primordial que a equipe multidisciplinar possua conhecimento e um olhar assertivo sobre as manifestações clínicas que indiquem coagulação, dessa forma, medidas efetivas poderão ser tomadas a fim de contribuir para um melhor prognóstico para os pacientes.

Dessa forma, faz-se necessário estudos futuros acerca da relação entre a COVID-19 e o AVE, visto que os pacientes acometidos, obtiveram maior gravidade e prognósticos desfavoráveis na afecção. Assim, é de fundamental relevância a realização de mais investigações, para que haja uma análise mais profunda acerca de tal temática. Portanto, com a realização de futuros trabalhos pela comunidade científica, os danos neurológicos serão mais averiguados e o conhecimento será gerado para um melhor manejo clínico aos pacientes acometidos pelas afecções neurológicas com COVID-19.

\section{Referências}

Al-ani, F., \& Lazo-langner, A. (2020). Thrombosis risk associated with COVID-19 infection. A scoping review. Thrombosis Research, 192, 152-160.

Brasil. (2020). Coronavírus Brasil. https://covid.saude.gov.br/

Crossetti, M. da G. O. (2012). Revisão integrativa de Pesquisa na Enfermagen o Rigor Cientifico que lhe é Exigido. Rev Gaúcha Enferm,Porto Alegre (RS), 33(2)(2), 8-9. http://www.scielo.br/pdf/rgenf/v33n2/01.pdf

Ferrarese, C., Silani, V., Priori, A., Galimberti, S., Agostoni, E., Monaco, S., Padovani, A., \& Tedeschi, G. (2020). An Italian multicenter retrospectiveprospective observational study on neurological manifestations of COVID-19 (NEUROCOVID). Neurological Sciences, 41(6), 1355-1359. https://doi.org/10.1007/s10072-020-04450-1

Ghannam, M., Alshaer, Q., Al-Chalabi, M., Zakarna, L., Robertson, J., \& Manousakis, G. (2020). Neurological involvement of coronavirus disease 2019: a systematic review. Journal of Neurology, 1-19. https://doi.org/10.1007/s00415-020-09990-2

Leonardi, M., Padovani, A., \& McArthur, J. C. (2020). Neurological manifestations associated with COVID-19: a review and a call for action. Journal of Neurology, 267(6), 1573-1576. https://doi.org/10.1007/s00415-020-09896-z

Lin, C., Arevalo, Y. A., Nanavati, H. D., \& Lin, D. M. (2020). Racial differences and an increased systemic inflammatory response are seen in patients with COVID-19 and ischemic stroke. Brain, Behavior, \& Immunity - Health, 8(July), 100137. https://doi.org/10.1016/j.bbih.2020.100137

Montalvan, V., Lee, J., Bueso, T., De Toledo, J., \& Rivas, K. (2020). Neurological manifestations of COVID-19 and other coronavirus infections: A systematic review. Clinical Neurology and Neurosurgery, 194, 105921. https://doi.org/10.1016/j.clineuro.2020.105921 
Research, Society and Development, v. 10, n. 1, e19610111535, 2021

(CC BY 4.0) | ISSN 2525-3409 | DOI: http://dx.doi.org/10.33448/rsd-v10i1.11535

Nepal, G., Rehrig, J. H., Shrestha, G. S., Shing, Y. K., Yadav, J. K., Ojha, R., Pokhrel, G., Tu, Z. L., \& Huang, D. Y. (2020). Neurological manifestations of COVID-19: A systematic review. Critical Care, 24(1), 1-11. https://doi.org/10.1186/s13054-020-03121-z

Opas. (2020). Folha Informativa COVID-19 - Escritório da OPAS e da OMS no Brasil. https:// www.paho.org/pt/covid19

Ortiz, M., Valencia, N., Moreno, E., Zafra, M., Espinel, L., Villarreal, D., Hernández, L., Caro, P., Galindo, M., Sierra, V., Peña, A., Gutiérrez, M., \& Bayona, H. (2020). ACV y covid-19: una revisión de los estudios observacionales publicados en época de pandemia. Acta Neurológica Colombiana, 36(2), 63-74. https://doi.org/10.22379/24224022280

Pereira, A. S., Shitsuka, D. M., Parreira, F. J., \& Shitsuka, R. (2018). Metodologia da pesquisa científica. In Metodologia da Pesquisa Científica. https://repositorio.ufsm.br/bitstream/handle/1/15824/Lic_Computacao_Metodologia-Pesquisa-Cientifica.pdf?sequence=1.

Pranata, R., Huang, I., Lim, M. A., Wahjoepramono, E. J., \& July, J. (2020). Impact of cerebrovascular and cardiovascular diseases on mort ality and severity of COVID-19-systematic review, meta-analysis, and meta-regression. Journal of Stroke and Cerebrovascular Diseases : The Official Journal of National Stroke Association, 29(8), 104949. https://doi.org/10.1016/j.jstrokecerebrovasdis.2020.104949

Tan, Y.-K., Goh, C., Leow, A. S. T., Tambyah, P. A., Ang, A., Yap, E.-S., Tu, T.-M., Sharma, V. K., Yeo, L. L. L., Chan, B. P. L., \& Tan, B. Y. Q. (2020). COVID-19 and ischemic stroke: a systematic review and meta-summary of the literature. Journal of Thrombosis and Thrombolysis, 50(3), 587-595. https://doi.org/10.1007/s11239-020-02228-y

Violi, F., Pastori, D., Cangemi, R., Pignatelli, P., \& Loffredo, L. (2020). Hypercoagulation and Antithrombotic Treatment in Coronavirus 2019: A New Challenge. Thrombosis and Haemostasis, 120(6), 949-956. https://doi.org/10.1055/s-0040-1710317

Whittaker, A., Anson, M., \& Harky, A. (2020). Neurological Manifestations of COVID-19: A systematic review and current update. Acta Neurologica Scandinavica, 142(1), 14-22. https://doi.org/10.1111/ane.13266 